\title{
Preliminary study of ultrasonic transducer characteristic on the breast surface
}

\begin{abstract}
This paper presents the initial result of an investigation on the application of ultrasonic sensors for a breast surface mapping. Ultrasonic transmitter and receiver were used to perceive the objects by emitting a short ultrasonic burst. The application for this system will be used in ultrasound tomography. The purpose of this study is to investigate the usability of the low frequency ultrasonic transducer to be applied in the medical field. FPGA technology and data acquisition system were used to establish the embedded system of the ultrasound tomography. FPGA is used to generate a pulse by the transmitter and the pulse is set perpendicular to the breast surface. The signal that has been reflected from the breast surface will be captured by the receiver. The results obtained shows that the system is capable of detecting the object. We also managed to demonstrate the noise reduction technique done in the frequency domain to suppress the unwanted signal.
\end{abstract}

Keyword: Ultrasonic transducer; Ultrasound; FPGA 